\title{
A NEW CALCULATION OF ATMOSPHERIC NEUTRINO FLUXES
}

\author{
D.H. Perkins \\ Nuclear Physics Laboratory, University of Oxford
}

\begin{abstract}
Summary: Atmospheric neutrino fluxes have been computed directly from recent measurements of muon momentum spectra in the stratosphere. The results agree within $15 \%$ with the previous calculations by Barr, Gaisser and Stanev by a different method, although they predict a somewhat different variation of flux with geomagnetic latitude. These results do not support the suggestion that the atmospheric neutrino anomaly can be interpreted as due to excess electron events from proton decay $p \rightarrow e^{+} \nu \nu$.
\end{abstract}

\section{Introduction}

The interpretation of the anomalous ratio $\nu_{\mu} / \nu_{e}$ of contained events due to atmospheric neutrinos observed in underground experiments (see Table 1) has been beset by uncertainties in the calculated absolute values of neutrino fluxes. It should be emphasized that although absolute fluxes may be uncertain, the calculated flux ratio $\left(\phi\left(\nu_{\mu}\right) / \phi\left(\nu_{e}\right) \simeq 2.1\right.$ for $\left.E_{\nu} \leq 1 \mathrm{GeV}\right)$ is stable, being almost entirely dependent on the kinematics of pion and muon decay, and not on the details of energy spectra.

The customary approach in calculating the neutrino fluxes has been to use the measured spectra of primary cosmic rays - protons, helium and heavier nuclei - together with data from accelerator experiments, to compute the spectra of the parent pions and muons generated via the nuclear cascades produced by the primaries as they traverse the atmosphere. Table 2 shows total numbers of single track muon and electron events in the Kamioka detector predicted by various calculations ${ }^{(1,2,3,4)}$ along these lines, together with the observed numbers ${ }^{(5)}$. The discrepancies at low energies are particularly spectacular: for $0.2-0.3 \mathrm{GeV}$ neutrino energy, for example, the rates predicted by Bugaev and Naumov ${ }^{(4)}$ are about a factor two less than those of Barr, Gaisser and Stanev ${ }^{(1)}$. This makes it difficult to know whether, for example, the observed anomalous value of the $\nu_{\mu} / \nu_{e}$ ratio is due to a deficit of muon events arising from oscillation of $\nu_{\mu}$ to another neutrino flavour, or to an excess of electron events associated with an unusual mode of proton decay, $p \rightarrow e^{+} \nu \nu$, as suggested by Mann, Kafka and Leeson ${ }^{(6)}$.

In this paper, after some comments on the possible origin of these discrepancies, a new and independent calculation of neutrino fluxes is presented which is deduced entirely from recently measured muon spectra at high altitude, thus circumventing many of the uncertainties in the conventional approach.

\section{Problems for existing flux calculations}

What is at issue here is the calculation of the atmospheric spectrum of pions (and to a lesser extent kaons), which upon decay give rise to the muons 
and neutrinos. The calculated pion spectra depend upon an integration over the primary spectrum of the distribution $d N / d x$ in the ratio $x=E_{\pi} / E_{0}$ of the pion to the proton (or nucleon) energy. The proton energies of importance for the present problem are in the range $0-30 \mathrm{GeV}$. Unfortunately, accelerator data on $d N / d x$ exist at very few values of the incident energy, and even then are incomplete, the measured cross-sections covering only a part of the range in $p_{T}$ or $x$ or both. For an $x$ distribution scaling with primary energy, and for a differential primary energy spectrum in the form of a power law, $E^{-\gamma} d E$, where $\gamma \simeq 2.7$, the production spectrum of pions will follow a similar power law, with a weighting factor $Z=\int_{0}^{1} x^{\gamma-1}(d N / d x) d x$. In this case the exact form of $d N / d x$ at very small $x$ may not be too important (it has approximately the form $\left.e^{-k x}\right) .^{(22)}$ Unfortunately, the primary spectrum in the energy region below $20 \mathrm{GeV}$ deviates substantially from a simple power law, particularly at low geomagnetic latitudes (the effective rigidity cut-off at the equator is 15 GV), and also at times of maximum solar activity. Furthermore, as pointed out below, the form of $d N / d x$ is energy-dependent. Thus the exact form of $d N / d x$ and its energy dependence can have a major effect on the absolute values of the neutrino fluxes below 1 or $2 \mathrm{GeV}$, and particularly on the variation of flux with magnetic latitude and the level of solar activity. The discrepancies between different calculations shown in Table 2 are therefore hardly surprising, especially when one takes into account that there are considerable variations - - even as large as a factor 2 - in measured values of the primary fluxes.

Recently, Gaisser ${ }^{(7)}$ has suggested that the discrepancies in the calculations originate primarily in the different values assumed for the pion distributions $d N / d x$ at small $x$. Fig 1 shows the quantity $x d N / d x$ for the sum of positive and negative pions at $E_{o}=24 \mathrm{GeV}$, where the most complete data is available. They have been calculated by integrating over transverse momentum the published values of the Lorentz-invariant cross-section $E d^{3} \sigma / d p^{3}$ measured in p-Be and $\mathrm{p}-\mathrm{Al}$ collisions for $x \geq 0.167$ by Eichten et al ${ }^{\left({ }^{(8)}\right.}$, and for $\mathrm{p}$-p collisions in a bubble chamber experiment by Mück et al ${ }^{(9)}$. These last cover smaller $x$ values but do involve some extrapolations at low transverse momentum, $p_{T}$. The larger intensities obtained for the nuclear targets are expected: aside from this difference of 15 or $20 \%$, the two experiments are compatible in the region where they overlap. The histogram and dotted curve show, respectively, the predictions of the models of Barr et al and of Bugaev and Naumov ${ }^{(7)}$. The latter apparently gives a better representation of the shape of $x d N / d x$ for this particular case. Fig 1 also includes the data of Mück et al for $E_{o}=12 \mathrm{GeV}$, showing that scaling (independence of $d N / d x$ on $E_{o}$ ) does not hold.

\section{High Altitude Muon Data}

A more direct approach to the question of neutrino fluxes, and one long advocated by the author ${ }^{(10)}$, is to exploit direct measurements of muon fluxes in the atmosphere, as was first done by Tam and Young ${ }^{(11)}$, following the work in 1954 of Olbert ${ }^{(12)}$, who deduced pion fluxes from the muon flux measurements at high altitude taken in 1948 and published in 1950 by Conversi ${ }^{(13)}$. Very simply, over $90 \%$ of contained $\nu_{e}$ and $\nu_{\mu}$ events are produced by neutrinos from pion and muon decay. There is an obvious correspondence between the momentum 
distributions of $\nu_{\mu}$ and $\mu$ from pion decay, and of course the $\nu_{\mu}$ and $\nu_{e}$ spectra from muon decay are readily calculated when the muon flux is known.

The first high altitude $\mu^{+}$and $\mu^{-}$fluxes were measured by Conversi, using Geiger counters and absorbers, and were restricted to a single momentum interval $\left(p_{\mu}=315-348 \mathrm{MeV} / \mathrm{c}\right)$ and to aircraft altitudes, that is atmospheric depths of $X \geq 300 \mathrm{~g} \mathrm{~cm}^{-2}$. This provided insufficient information to reliably parameterise the pion spectrum, especially at low momenta $\left(p_{\pi}<0.5 \mathrm{GeV} / \mathrm{c}\right)$. Recently, the situation has been completely transformed however by new measurements performed by the MASS collaboration (Circella et al ${ }^{(14)}$ ), of muon spectra measured with the help of a superconducting magnet carried on a balloon flight from Prince Albert, Saskatchewan, during 1989. So far, only negative muon data are available, but they cover the momentum range $0.3-10 \mathrm{GeV} / \mathrm{c}$ and atmospheric depths $X=11,37,72,112,163,283$ and $615 \mathrm{~g} \mathrm{~cm}^{-2}$. Figs 2 and 3 show the Conversi as well as the MASS data, both taken at times near to solar maximum (in 1948 and 1989 respectively).

In order to compute neutrino and muon fluxes associated with pion and muon decay, we adopt a trial pion production spectrum, assumed to have a momentum dependence independent of atmospheric depth, with empirical parameterisation chosen to fit the muon data. A pion production rate in the vertical direction of the following form was assumed (units are numbers of $\pi^{+}$ and $\left.\pi^{-} \mathrm{g}^{-1} \mathrm{~cm}^{2} \mathrm{~s}^{-1} \mathrm{st}^{-1} \mathrm{GeV} / \mathrm{c}^{-1}\right)$ :-

$$
\frac{d^{2} N_{\pi}}{d X d p}=\frac{A}{D} e^{-X / D} \frac{1}{(1+b / p)^{4}} \frac{1}{p^{2.7}} f(p, \lambda)
$$

where $X$ is the atmospheric depth in $\mathrm{g} \mathrm{cm}^{-2}, p$ is the pion momentum in $\mathrm{GeV} / \mathrm{c}$, and $b=0.25 \mathrm{GeV} / \mathrm{c} . D=135 \mathrm{~g} \mathrm{~cm}^{-2}$ is the attenuation length of the assumed exponential absorption of the pion-producing radiation, and the best fitting value of $A=0.25$. The power law dependence at high momentum is chosen to fit muon spectrometer data at sea level. The factor $f(p, \lambda)$ takes account of dependence on the magnetic latitude $\lambda$. On the basis of the Conversi measurements of the latitude effect discussed later, we take the empirical form

$$
\begin{aligned}
f(p, \lambda) & =(p / 6.0)^{\alpha(\lambda)} & & \text { for } p \leq 6 \mathrm{GeV} / \mathrm{c} \\
& =1.0 & & \text { for } p>6 \mathrm{GeV} / \mathrm{c}
\end{aligned}
$$

where

$$
\alpha(\lambda)=0.35 \cos ^{4} \lambda-0.017
$$

With this parameterisation, $\alpha(\lambda)=0$ at $\lambda=62^{\circ}$, the latitude at which the MASS measurements were taken. The value of $\alpha(\lambda)$ was taken as zero for $\lambda>62^{\circ}$.

From formula (1), the vertical muon flux for $\lambda=62^{\circ}$ and $p_{\mu}=300-400$ $\mathrm{MeV} / \mathrm{c}$ has been calculated as a function of altitude. A Monte Carlo program was employed to generate a vertical pion production spectrum based on (1), and to follow the pions and decay muons through the atmosphere in small steps, taking account of ionisation energy losses and nuclear absorption, with the result shown by the upper curve in Fig 2, to be compared with the experimental results 
of the MASS group, as well as those of Conversi and the spectrometer fluxes at sea-level measured by Rastin ${ }^{(15)}$ at Nottingham. All numbers refer to negative muon fluxes. Negative muon fluxes from the Conversi and Rastin experiments and from the above formula were obtained by assuming a constant $\mu^{+} / \mu^{-}$ratio of 1.25 .

The Conversi results refer to magnetic latitude $50^{\circ}$ while the MASS spectra are for $62^{\circ}$. Taking into account also that the experiments were done near different solar maxima, precise agreement is hardly to be expected. Nevertheless, the Conversi data appear to be remarkably close to the more recent and more comprehensive measurements.

Fig 2 also shows the integral MASS muon fluxes for $p_{\mu} \geq 0.30 \mathrm{GeV} / \mathrm{c}$, together with the Rastin sea-level flux and the flux computed using the above formula. Finally, Fig 3 shows the muon momentum spectra at a few representative altitudes from the MASS experiment, together with the predictions from (1). Taken together with the results of Fig 2, the empirical formula appears to give a satisfactory representation of the new muon data. Clearly the parametric form assumed here for the pion spectrum is not unique, and the experimental errors in the muon data allow some variation in the constants employed. The representation of the depth dependence of pion production by a single attenuation length $D$ is clearly an over-simplification, which will doubtless need to be modified when more accurate data become available. For example, Thompson and Whalley ${ }^{(16)}$ estimate that some $4 \%$ of the muon flux at sea- level arises from decay of pions produced in secondary pion-mucleon collisions.

\section{Neutrino Flux Calculations}

The values of the atmospheric neutrino fluxes from pion and muon decay have been computed from (1) and (2) using the same program that generated the muon fluxes. The calculation has been done in several stages:-

\section{(a) Direct calculation of muon neutrino flux from pion decay}

Firstly, in order to check the validity of the $\mathrm{MC}$ computation, a calculation of vertical neutrino flux from pion decay only was made directly from the MASS spectrum, without any reference to an empirical pion spectrum. For values of $X \leq 35 \mathrm{~g} \mathrm{~cm}^{-2}$, the mean ionisation energy loss of vertical muons created at smaller $X$ values is less than $10 \%$ of their recorded momentum $(\geq 300 \mathrm{MeV} / \mathrm{c}$ in the MASS experiment), and in a first approximation can be neglected. Since $X \ll D$, the rate of generation of muons is practically independent of $X$. Assuming also that the pion parents have $\beta \simeq 1$, the vertical downward spectrum of muon neutrinos at sea level from pion decay, $h_{\nu}(E)$, is related to the vertical muon momentum spectrum at altitude by the infinite series

$$
h_{\nu}(E)=\frac{D}{X R} \sum_{i=1}^{\infty} \frac{r^{(i-1)} g_{\mu}\left(X, p_{i}\right)}{P_{\mu}\left(X, p_{i}\right)}
$$

where $E$ is the neutrino energy, $R=0.44$ is the assumed fraction of negative muons and $g_{\mu}\left(X, p_{i}\right)$ is the negative muon flux at depth $X$ and at momentum 
$p_{i}=r^{(i-1)} E / a$, where $a=1-m_{\mu}^{2} / m_{\pi}^{2}=0.427$ and $r=(1-a)^{-1}=1.745$. Thus $p_{1}=2.34 E, p_{2}=4.09 E$ etc. The quantity $P_{\mu}$ is the probability that a muon, created at any value $X^{\prime}<X$ will survive to $X$ without decaying

$$
P_{\mu}\left(X, p_{\mu}\right)=\frac{1}{(1+\alpha)}[1-\alpha X / 2 D(\alpha+2)+\ldots]
$$

where $\alpha$ is the ratio of the atmospheric scale height $(H=6.5 \mathrm{~km}$ in the upper atmosphere) to the muon decay length, $\left(p_{\mu} / m_{\mu} c\right) c \tau_{\mu}$. In numbers, $\alpha=1.04 / p_{\mu}$ where $p_{\mu}$ is the muon momentum in $\mathrm{GeV} / \mathrm{c}$. The correction terms included in brackets in (4) are always less than $5 \%$ for the chosen conditions (muons of $X \leq 37 \mathrm{~g} \mathrm{~cm}^{-2}, p_{\mu} \geq 0.3 \mathrm{GeV} / \mathrm{c}$ ).

The values of the muon spectra measured at $X=11 \mathrm{gcm}^{-2}$ and $X=$ $37 \mathrm{gcm}^{-2}$ were combined and applied to deduce the vertical $\left(\nu_{\mu}+\bar{\nu}_{\mu}\right)$ spectrum from pion decay, using (3) and (4), with the result shown by the points in Fig 4. The estimated statistical errors are shown, but it should be noted that there is some correlation between adjacent points. Systematic errors which result from truncating the sum (3) where the muon data runs out, are $5 \%$ or less. The corresponding spectrum of vertically downward muon neutrinos from pion decay, calculated from (1) and the Monte Carlo program, is given by the curves, which show the effect of a $\pm 10 \%$ variation in the parameter $b$ in (1). The two calculations are in fair agreement, suggesting that there are no gross errors in the Monte Carlo calculation or in the parameterisation of the pion spectrum. Note that this spot check of the neutrino flux, independent of pion spectrum parameterisation, holds only over the restricted energy interval $E_{\nu}=0.15-0.50$ $\mathrm{GeV}$. Fortunately, however, this is the most important region from the viewpoint of contained neutrino events.

\section{(b) The downward muon neutrino flux}

The next step in the Monte Carlo calculation is to compute the downward fluxes of $\nu_{\mu}$ and $\bar{\nu}_{\mu}$ from both pion and muon decay, taking account of muon polarisation effects and averaging over all zenith angles, $\theta$. For the latter purpose, a flat distribution was assumed over the range $\cos \theta=0.05-1.0$, the lower limit being an effective value imposed by the Earth's curvature, and the vertical depth $X$ in (1) was replaced by the slant depth $X \sec \theta$. These $\nu_{\mu}+\bar{\nu}_{\mu}$ fluxes are given in column 2 of Table 3 . They are the downward fluxes for $62^{\circ}$ latitude. They do not depend on the assumed latitude dependence in (2). They will be upper limits to the fluxes at solar maximum, averaged over both up and down zenith angles, since upward travelling neutrinos generally originate at lower latitudes and therefore will have a smaller flux.

\section{(c) Total neutrino fluxes}

The final part of the calculation, of up and down fluxes at arbitrary latitudes, involves the latitude dependence (2), as fitted to the Conversi muon data shown in Fig 5. This shows the dependence of flux on magnetic latitude, for muons at $X=300 \mathrm{~g} \mathrm{~cm}^{-2}$ and $p_{\mu}=315-348 \mathrm{MeV} / \mathrm{c}$, relative to the equator. 
The curve indicates the predicted variation from (1) and (2) for $X=300 \mathrm{~g} \mathrm{~cm}^{-2}$ and $p_{\mu}=300-400 \mathrm{MeV} / \mathrm{c}$. The chosen parameterisation is seen to be satisfactory: if anything, it slightly overestimates the magnitude of the latitude effect as observed by Conversi.

The fluxes of $\nu_{\mu}$ and $\nu_{e}$ and their antiparticles were computed from the parameterisations (1) and (2). Contributions due to $K^{ \pm, 0}$ decay were included, assuming $\left(K^{+}+K^{-}\right) /\left(\pi^{+}+\pi^{-}\right)=0.10$, equal numbers of charged and neutral kaons, and similar momentum spectra for kaons and pions. For neutrino energies $0.1-2 \mathrm{GeV}$, the liaon contribution to the neutrino flux averages $12 \%$, while that to the muon flux at high altitude $(X \sim D)$ in the corresponding range, $0.4-$ $4 \mathrm{GeV}$, averages $5 \%$. The difference between these figures was added to the neutrino fluxes calculated for pions alone. Table 3 shows the results for $\lambda=52^{\circ}$, compared with the results of Barr et al (1). In both cases, the fluxes refer to solar maximum. The fluxes calculated here are larger than those previously (10) estimated from the Conversi muon fluxes. The new, more detailed measurements at shallower depths in the atmosphere require a pion spectrum of considerably higher intensity at. low momentum.

We observe, firstly, that the fluxes calculated here for $\lambda=52^{\circ}$ are on average about $15 \%$ less than those of Barr et al, with no apparent energy dependence to the ratio. This rather good agreement is probably fortuitous, since Barr et al themselves assign a $20 \%$ error to their numbers, and the muon fluxes, upon which the present calculation is based, are stated ${ }^{(14)}$ to have a systematic uncertainty which could be as large as $20 \%$. Table 3 also shows the fluxes at $\lambda=0^{\circ}$. Here the discrepancy with the Barr et al fluxes is even smaller: on average, the flux calculated is about $2 \%$ larger than that of Barr et al. This apparently close agreement at two latitudes unfortunately however hides a fundamental difference in the two calculations: Barr et al predict a much stronger latitude dependence.

\section{Latitude Dependence}

Fig 5 shows, in addition to the Conversi muon data and the predicted variation of muon flux with magnetic latitude, results from the present calculation for downward-travelling neutrinos in the energy ranges $0.2-0.3$ and $0.8-1.0$ $\mathrm{GeV}$. The latitude dependence of downward neutrinos of $0.2-0.3 \mathrm{GeV}$ is almost the same as that of vertical muons of $0.3-0.4 \mathrm{GeV} / \mathrm{c}$ at $X=300 \mathrm{gcm}^{-2}$. The reason is that both originate from pions with about the same energy, as is obvious if one considers the kinematics of pi-mu decay and the typical ionisation energy loss of muons penetrating to $X=300 \mathrm{gcm}^{-2}$. Fig 6 shows the momentum spectrum of pions producing vertical muons of momentum $0.3-0.4 \mathrm{GeV} / \mathrm{c}$ at $300 \mathrm{~g} \mathrm{~cm}^{-2}$ depth, together with the pion spectrum responsible for downward neutrinos of $0.2-0.3 \mathrm{GeV}$ at sea level. The two spectra are quite similar, except that the pion spectrum responsible for the neutrinos has a high energy tail. So, we expect the latitude dependence of the downward neutrino flux to be similar to, but somewhat weaker than, that of the muons. The calculated variation between $52^{\circ}$ and $0^{\circ}$ is a factor 1.8 , compared with 2.0 for the muons. On the other hand, the upward flux of neutrinos varies rather little with latitude ( with roughly a $10 \%$ increase between $\lambda=0^{\circ}$ and $\lambda=50^{\circ}$ ), so that the latitude 
ratio of all neutrinos, up plus down, is reduced. The calculation for both up and down neutrinos predicts a factor 1.33 for $E_{\nu}=0.2-0.3 \mathrm{GeV}$, while the Barr et al paper gives a factor 1.74 , that is, as much as in the present calculation for the downward neutrinos alone. It seems difficult to understand this discrepancy if the Conversi latitude variation is taken at its face value. All these figures, it should be emphasised, refer to the maximum of the solar cycle.

\section{Conclusions}

The conclusions of this paper are as follows:-

1) An independent calculation of atmospheric neutrino fluxes at $52^{\circ}$ latitude has been made, based primarily on the measured momentum spectra of high altitude muons at $62^{\circ}$. The fluxes refer to solar maximum and the absolute values agree with those of Barr et al to within about $15 \%$. In particular, the ratio of fluxes in the two calculations appears to be a constant and energy-independent factor. This result does not support the calculations of Bugaev and Naumov, who predict considerably smaller fluxes, or the interpretation of the atmospheric neutrino anomaly in terms of an excess of electron events due to proton decay.

2) The latitude dependence of neutrino fluxes given here is based on the latitude variation of the muon intensity observed by Conversi. These muons would correspond roughly to neutrinos in the energy range $0.2-0.3 \mathrm{GeV}$, for which the calculated variation with magnetic latitude is smaller than that of Barr et al. Presumably this matter could be resolved by new measurements of high altitude muon spectra as a function of geomagnetic latitude. 


\section{References}

1. G. Barr, T.K. Gaisser and T. Stanev Phys. Rev. D39 (1989) 3532.

2. M. Honda et al Phys. Lett. B248 (1990) 193.

3. H. Lee and Y.S. Koh Nuov. Cim. 105B (1990) 883.

4. E.V. Bugaev and V.A. Naumov Phys. Lett. B232 (1989) 391.

5. T. Kajita Proc. XXVI Int. Conf. High Energy Phys. Dallas 1992 Vol 2, p.1187 (AIP Conf. Proc. No. 272).

6. W.A. Mann, T. Kafka and W. Leeson Phys. Lett. B291 (1992) 200.

7. T.K. Gaisser, private comm. and Proc. Atmospheric Neutrino Workshop, 23rd Int. Conf. on Cosmic Rays, Calgary (July 1993).

8. T. Eichten et al Nucl. Phys. B44 (1972) 333.

9. H.J. Mück et al Phys. Lett. 39B (1972) 303.

10. D.H. Perkins "Atmospheric Neutrino Flux" Oxford report OUNP 85/84 (1984): see also Nucl. Phys. B399 (1993) 3.

11. A.C. Tam and E.C.M. Young; Acta Phys. Acad. Sci. Hungary Suppl. 4 (1970) 307.

12. S. Olbert Phys. Rev. 96 (1954) 1410.

13. M. Conversi Phys. Rev. 79 (1950) 749.

14. M. Circella et al "Cosmic Ray Muon Spectrum in the Atmosphere" Proc. 23rd Int. Conf. on Cosmic Rays, Calgary (July 1993); see also Frascati preprint LNF-93/055(R), p.11.

15. B.C. Rastin J. Phys. G10 (1984) 1609.

16. M.G. Thomson and M.R. Whalley J. Phys. G3 (1977) 97.

17. K.S. Hirata et al Phys. Lett. B280 (1992) 146.

18. D. Casper et al Phys. Rev. Lett. 66 (1991) 2561.

19. O. Perdereau et al Proc. of Moriond Conf. 1991 on Tests of Fundamental Conservation Laws in Physics.

20. M. Aglietta et al Europhys. Lett. 8 (1989) 611.

21. P.J. Litchfield Proc. of EPS Conf. on High Energy Physics, Marseilles (July 1993).

22. G. Cocconi, L. Koester and D.H. Perkins; Berkeley High Energy Physics Study, UCRL-10022 p.167 (1961). 
Table 1: Contained event $\mu$ to $e$ ratios

\begin{tabular}{lcc} 
Experiment & Kiton yr & $(\mu / e)_{\text {obs }} /(\mu / e)_{\text {calc }}$ \\
\hline Kamioka $^{(17)}$ & 6.1 & $0.60 \pm 0.07$ \\
IMB $^{(18)}$ & 7.7 & $0.54 \pm 0.07$ \\
Frejus $^{(19)}$ & 1.56 & $0.87 \pm 0.21$ \\
Nusex $^{(20)}$ & 0.40 & $0.99 \pm 0.40$ \\
Sourlan 2 $^{(21)}$ & 1.0 & $0.69 \pm 0.19$
\end{tabular}

Table 2: Contained event rates for Kamioka $4.92 \mathrm{Kton}$ year

\begin{tabular}{lccccc} 
Events & Data $^{(5)}$ & \multicolumn{4}{c}{ Monte Carlo predictions } \\
& & BGS $^{(1)}$ & HK $^{(2)}$ & LK $^{(3)}$ & BN $^{(4)}$ \\
\hline$\mu$-like & 151 & 261 & 234 & 205 & 179 \\
$e$-like & 159 & 165 & 146 & 128 & 100 \\
\hline Total & 310 & 426 & 380 & 333 & 279 \\
\hline
\end{tabular}




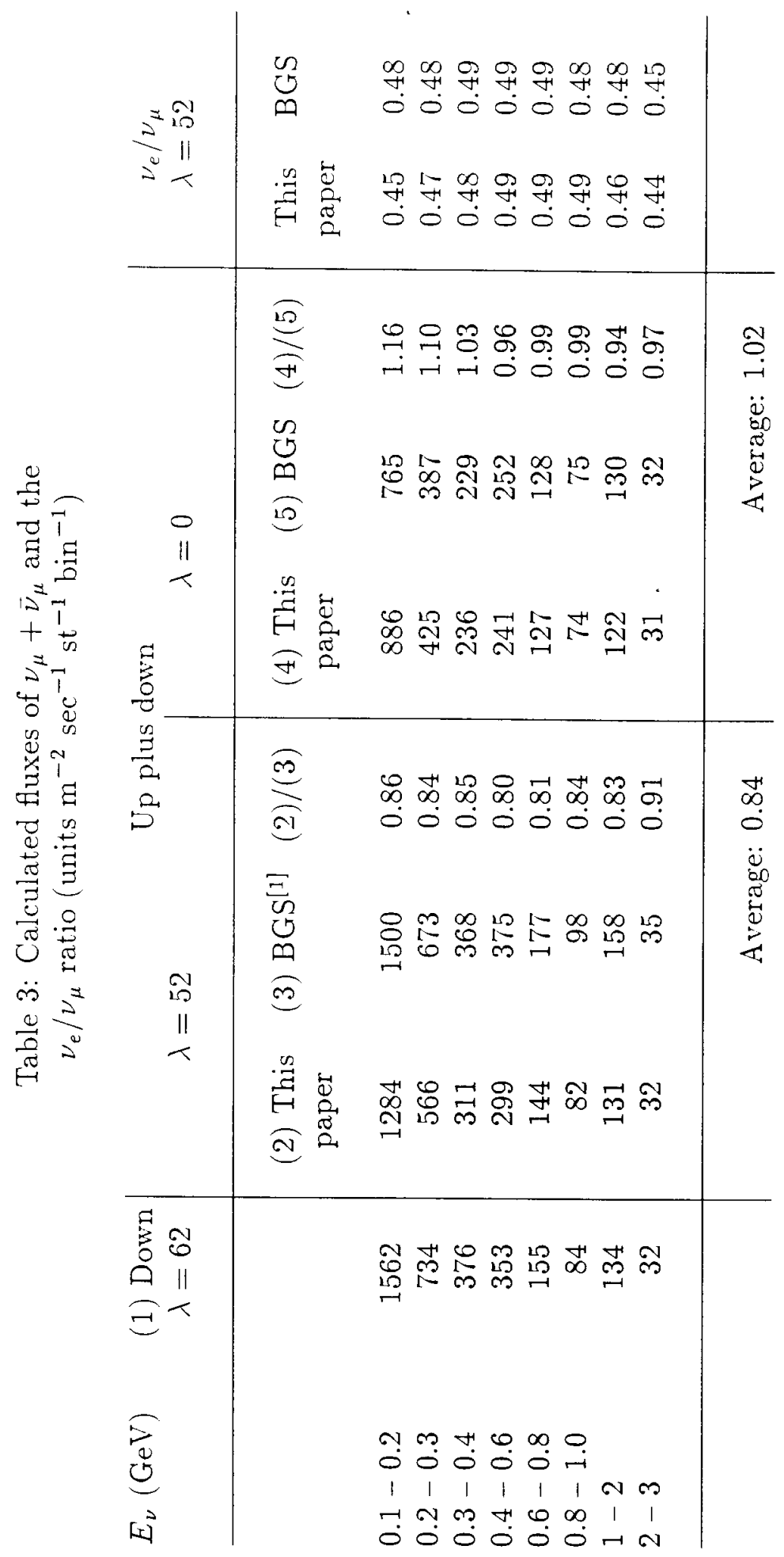




\section{Figure Captions}

Fig. $1 \times$ distribution of positive plus negative pions observed in $\mathrm{pBe}$ and $\mathrm{pAl}$ collisions at proton energy $E_{0}=24 \mathrm{GeV}$ by Eichten et al ${ }^{(8)}$ and in $\mathrm{pp}$ collisions at $E_{0}=24 \mathrm{GeV}$ and $E_{0}=12 \mathrm{GeV}$ by Mück et al ${ }^{(9)}$. The histogram and dashed curve show, respectively, the predictions of the $\mathrm{MC}$ program used by Gaisser et al ${ }^{(7)}$ and Bugaev and Naumov ${ }^{(4)}$, at $E_{0}=$ $24 \mathrm{GeV}$.

Fig.2 Vertical negative muon fluxes as a function of depth in the atmosphere. The upper set of points are for muons of momentum $\approx 0.3-0.4 \mathrm{GeV} / \mathrm{c}$, as measured in a balloon by Circella et al ${ }^{(14)}$, in aircraft flights by Conversi ${ }^{(13)}$ and at sea-level by Rastin ${ }^{(15)}$. The lower set of points shows the integral muon fluxes for $p_{\mu}>0.3 \mathrm{GeV} / \mathrm{c}$ measured by Circella et al. The curves in both cases were computed using the pion spectrum in equation (1).

Fig.3 Examples of momentum spectra of negative muons at atmospheric depths $X=37,112,163$ and $1030 \mathrm{gcm}^{-2}$. The experimental points are from the work of Circella et $a l^{(14)}$ at altitude and from that of Rastin ${ }^{(15)}$ at sea-level. The curves were calculated from equation (1).

Fig.4 Vertical flux of $\nu_{\mu}$ and $\bar{\nu}_{\mu}$ from pion decay only. The points with error bars were computed from the high altitude muon data of Figs. 2 and 3 , using equations (3) and (4). The curves show the prediction from the pion spectrum in equation (1), for three values of the parameter $b=0.225,0.25$ and 0.275 .

Fig.5 Magnetic latitude dependence of vertical muon flux, relative to equator, measured by Conversi ${ }^{(13)}$ for $p_{\mu}=0.315-0.348 \mathrm{GeV} / \mathrm{c}$ and atmospheric depth $X=300 \mathrm{gcm}^{-2}$. The solid curve shows the predictions from equations ( 1 ) and (2), for muons of $p_{\mu}=0.3-0.4 \mathrm{GeV} / \mathrm{c}$. The dashed curves (also computed from (1) and (2)) are for downward $\nu_{\mu}$ from pion and muon decay of $E_{\nu}=0.2-0.3 \mathrm{GeV}$ and $E_{\nu}=0.8-1.0 \mathrm{GeV}$.

Fig.6 Pion spectra responsible for muons and neutrinos, computed using equation (1). The dashed histogram is for pions producing muons of $p_{\mu}=$ $0.3-0.4 \mathrm{GeV} / \mathrm{c}$ at $X=300 \mathrm{gcm}^{-2}$, while the solid histogram is for $\nu_{\mu}$ of energy $E_{\nu}=0.2-0.3 \mathrm{GeV}$. 


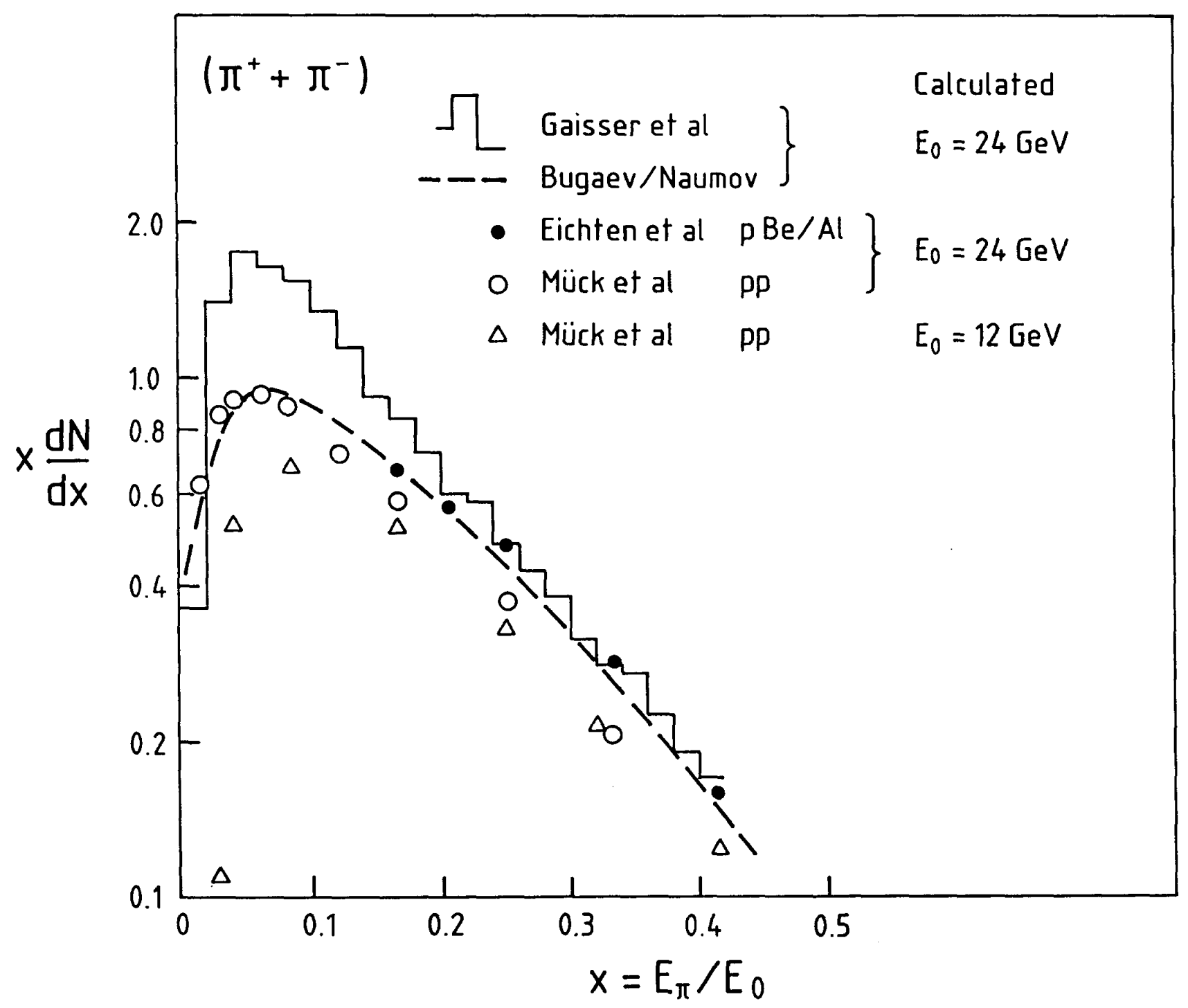

Fig. 1 


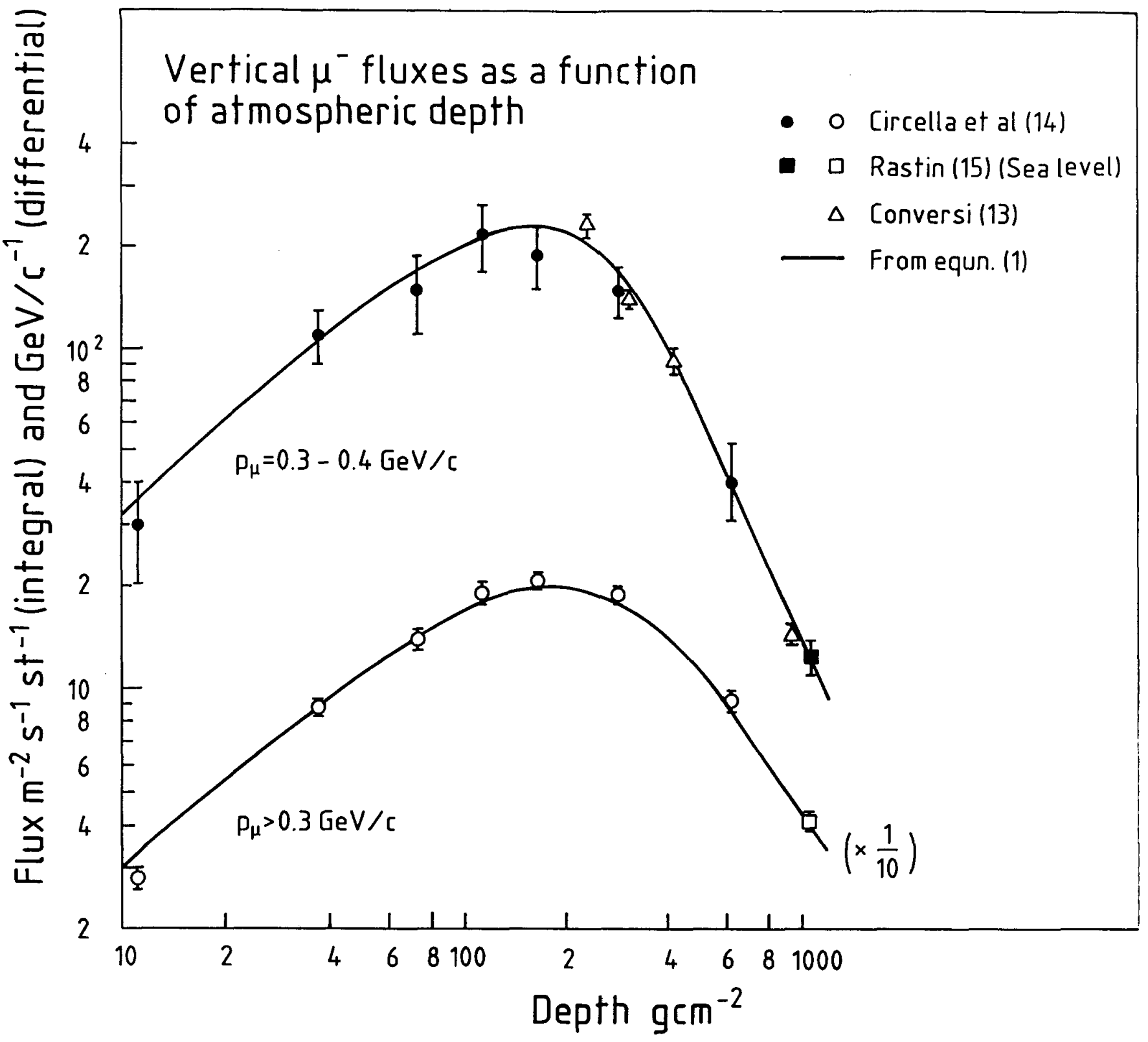

Fig. 2 


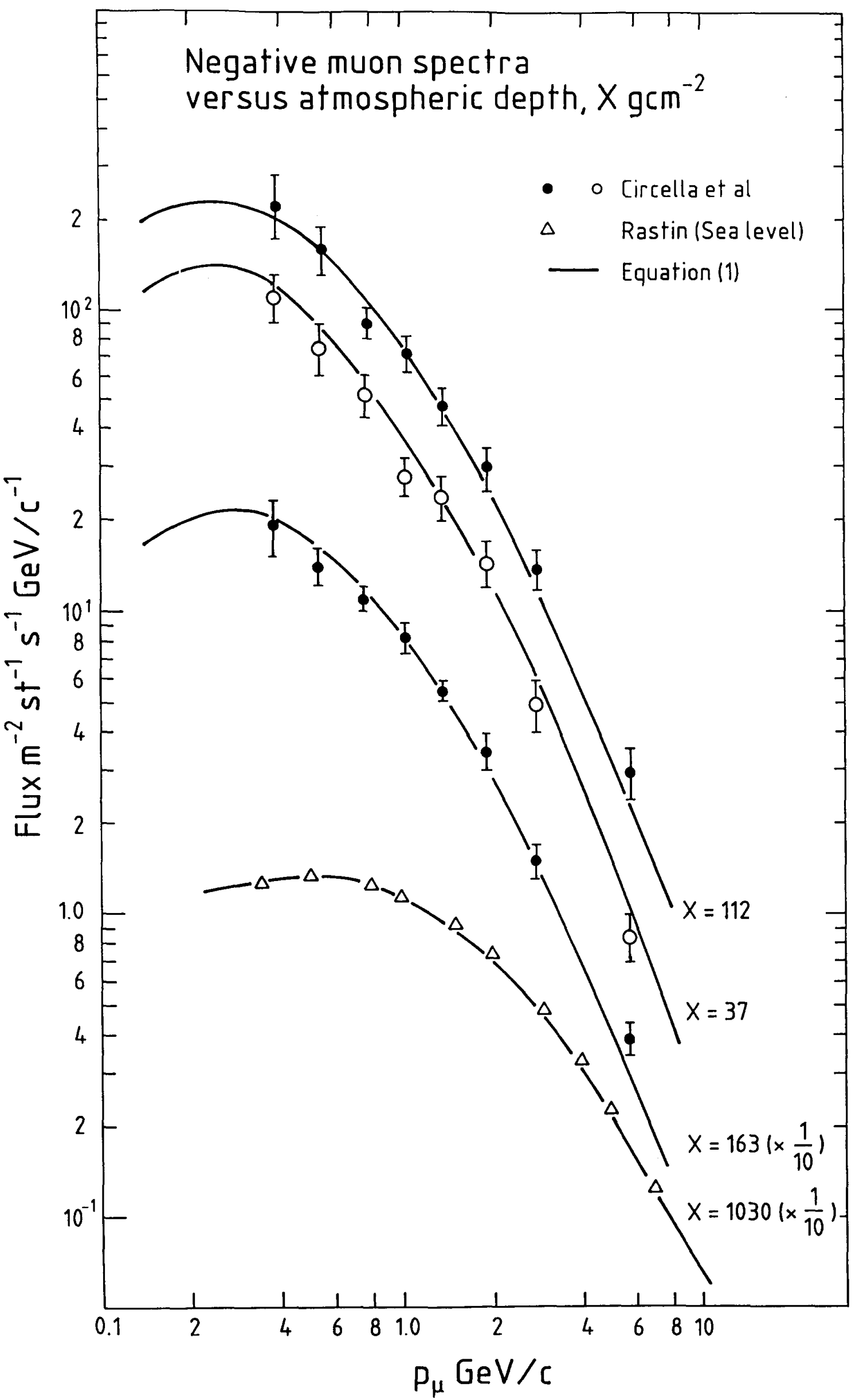

Fig. 3 


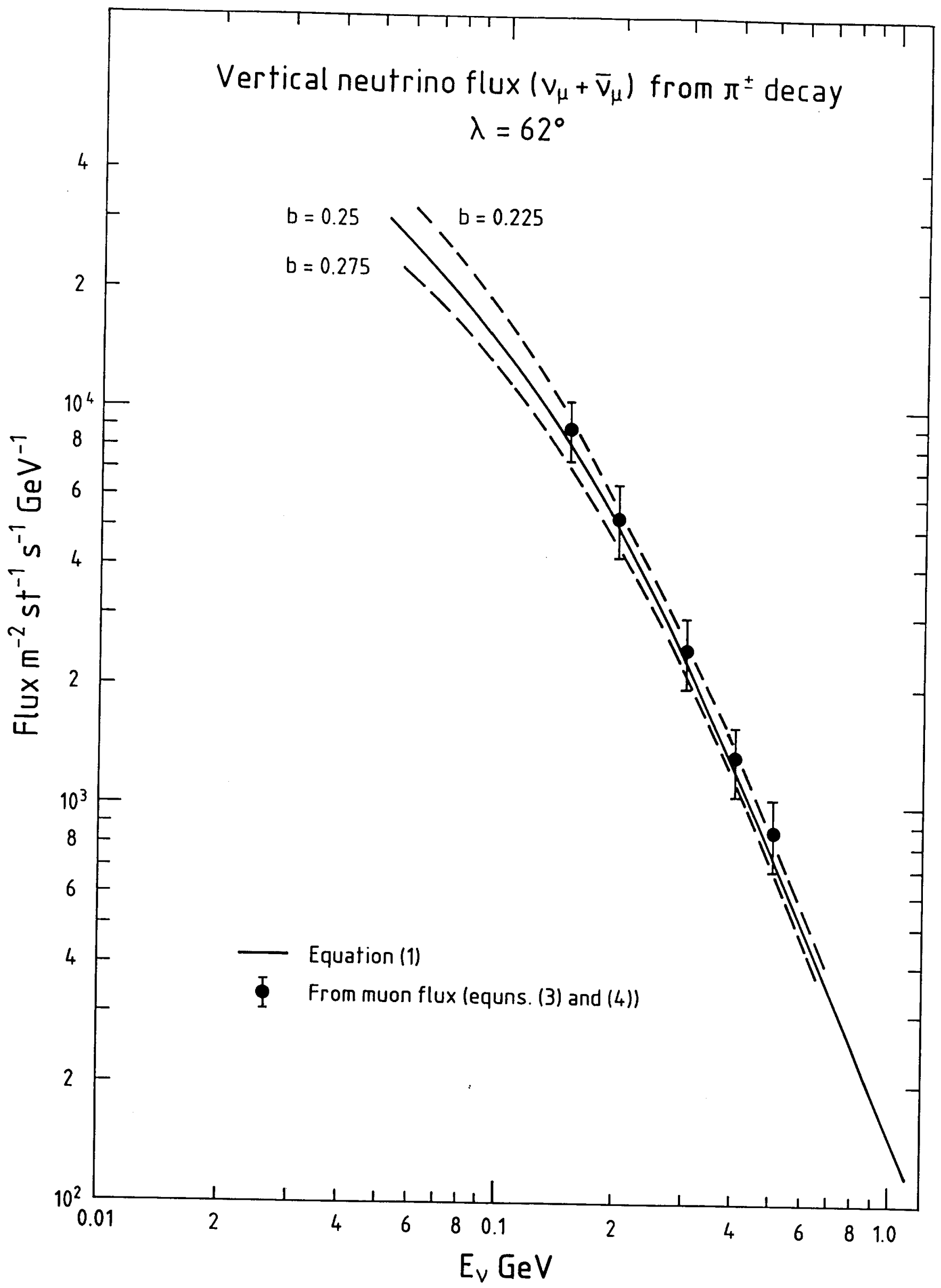

Fig. 4 


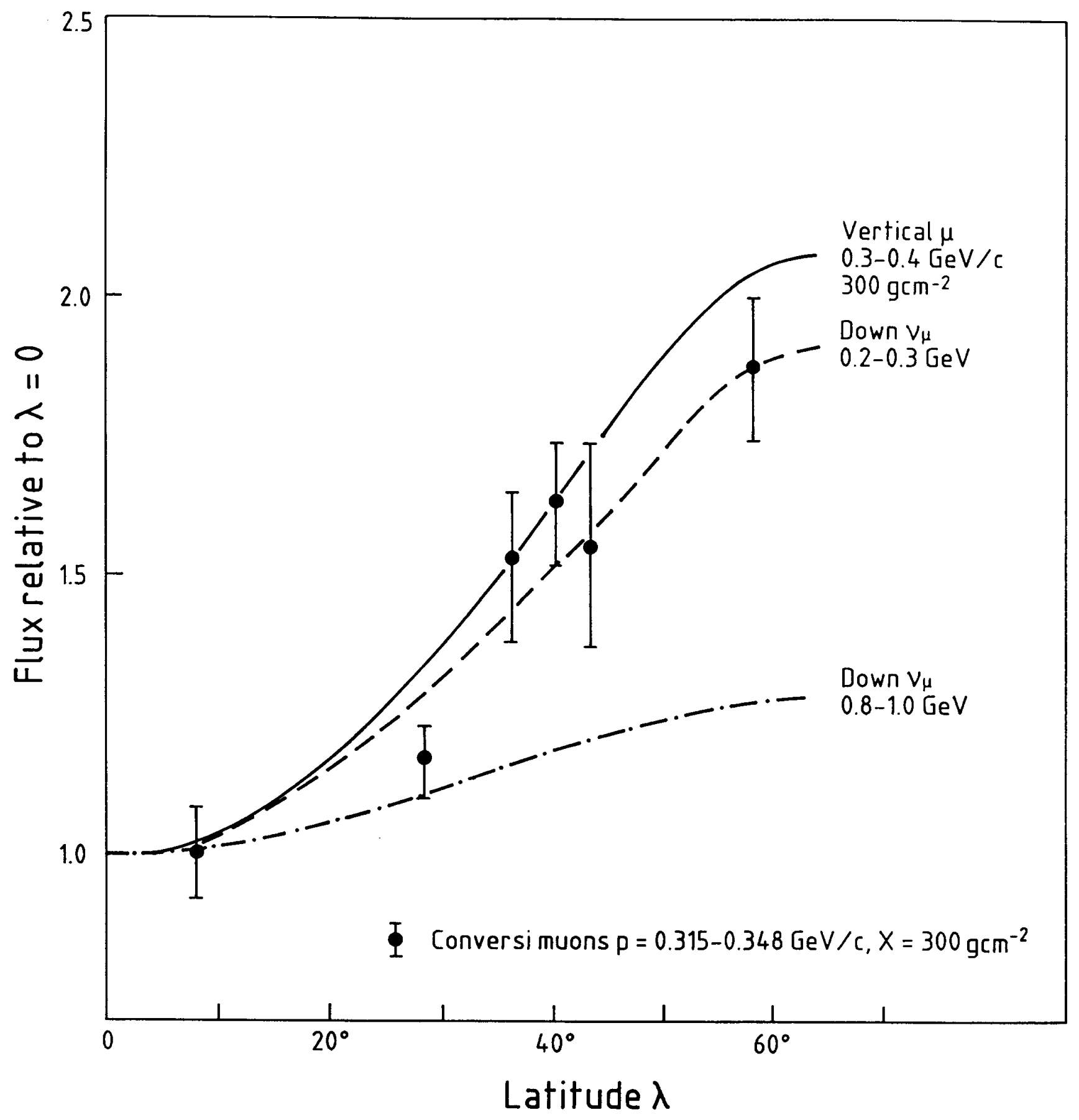

Fig. 5 


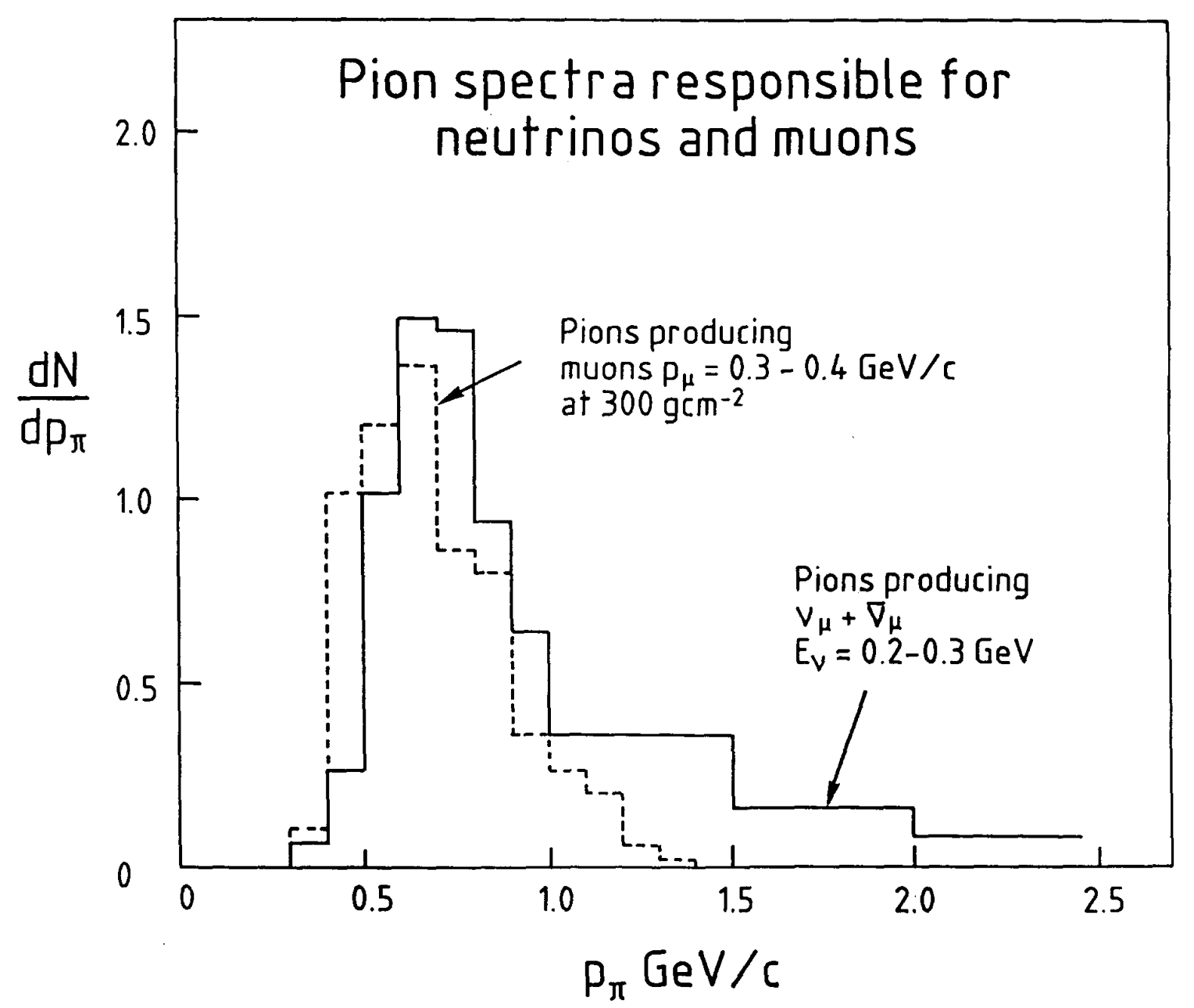

Fig. 6 
